\title{
The effect of a density difference on shear-layer instability
}

\author{
By ROBERT F. DAVEY AND ANATOL ROSHKO
}

California Institute of Technology, Pasadena

(Received 11 August 1971 and in revised form 30 December 1971)

Measurements of mass flow rate and mean density have been made in separated laminar boundary layers with large transverse density gradients. Two-dimensional shear layers were formed by exhausting a half-jet of one gas into a reservoir of another gas with a different molecular weight. Two freons with a density ratio of 1.98 and unusual properties which permitted the measurement of the mass flow rate with a single hot wire were used. An analysis of the mass flow rate fluctuations showed that a negative density gradient (i.e. light gas flowing into heavy) increases the amplification rate of the instability oscillations and reduces the frequency and wavenumber. Opposite trends were observed when the density gradient was positive. These findings are in agreement with recent theoretical predictions.

\section{Introduction}

Interest in the stability of the separated boundary layer dates back almost a century to the work of Rayleigh. Only rather recently, however, has the problem been modelled with sufficient accuracy to bring the results of theoretical and experimental investigations into essential agreement. The results reported by Freymuth (1966) in particular indicate that, at least for the homogeneous incompressible case, the problem is now well in hand. An enlightening survey of the progress made in understanding the homogeneous free shear layer has been prepared recently by Michalke (1970).

Less progress has been reported in the development of an understanding of the effect of a difference in the densities of the two fluids on either side of a laminar mixing layer; most theoretical investigations have been devoted to examining the effect of buoyancy forces on stratified flows. Very few authors have discussed the case where the Froude number is large and the dominant heterogeneous effects are due to inertial forces produced by the density gradient. Gropengiesser (1969) analysed the stability of compressible shear layers for Mach numbers up to three and for several temperature ratios. His results were the first to indicate that a density gradient (induced by temperature differences) could increase the amplification rate of instability waves. Maslowe \& Kelly (1971) used a flow model proposed by Holmboe to examine the incompressible case and found that a density gradient either increases or reduces the disturbance amplification rate, depending upon its direction and magnitude. In particular, they found that an increase occurs when the density and velocity gradients are of opposite sign. 
The present research grew out of a long-term programme directed towards developing an understanding of separated flows in general and the turbulent mixing layer in particular. Spark shadowgraphs of rectangular and axisymmetric jets showed that a density heterogeniety could markedly affect the character of the transition to turbulence. Figure 1 (plate 1) contrasts shear layers formed by helium flowing into air and vice versa. With helium flowing into air the transition to turbulence occurs in a short distance and the mixing layer grows rapidly. In the second case the transition is more gradual and a pronounced wave-like structure is apparent. It was felt that the differences in transition characteristics were related to the stability properties of the laminar shear layer lying between the separation point and the beginning of the turbulent region. $†$ The present experiments were designed to study the stability characteristics with the following specific goals in mind. First, measurements of mean velocity and density were desired to study the streamwise flow development and for comparison with profiles used in theoretical analyses, particularly the Holmboe profiles used by Maslowe \& Kelly. Second, a determination of the disturbance amplification rate was sought to aid understanding of the beginnings of the jet transition phenomenon. Finally, measurements of the instability wave parameters including the frequency, wavelength, phase variation and the transverse distribution of the fluctuation amplitude were desired, especially after the computations of Maslowe \& Kelly became available, to provide a basis for comparison.

\section{Experimental equipment}

\subsection{Jet facility}

A two-dimensional shear layer with a large transverse density gradient was produced by exhausting one gas from a $\frac{3}{8}$ by $1 \frac{1}{2}$ in. rectangular half-jet into a sealed tank containing a second gas with a different molecular weight. The halfjet was formed at the exit of a two-dimensional circular-arc nozzle which contracted fifteen-to-one to the exit dimensions (figure 2). A slide valve installed across the nozzle exit kept the two gases separated until the flow was turned on. A relatively large settling chamber was placed upstream of the nozzle and screens and honeycomb sections were installed to reduce the turbulence level to less than $0 \cdot 1 \%$ of the jet velocity.

Gas was supplied from cylinders containing liquid freon; electrical heating tapes were used to replace the heat lost from boiling. The jet velocity was controlled by a pressure regulator and a sonic needle valve, and was measured by a Pitot tube placed at the nozzle exit.

The tank which contained the background gas was about $30 \mathrm{in}$. high and had a 15 in. diameter (figure 2). A two-directional traverse allowed the positioning of instruments to accuracies of \pm 0.001 in. A small fan was installed to blend the gas mixtures used for calibrating the density sensitive instruments and the tank was lined with polyurethane foam to reduce sound reflexions.

$\dagger$ In figure $1(b)$ most of the shear layer has already developed into a late nonlinear stage of instability. The linear region of exponentially growing disturbances extends, approximately, to the end of the first low amplitude wave. 


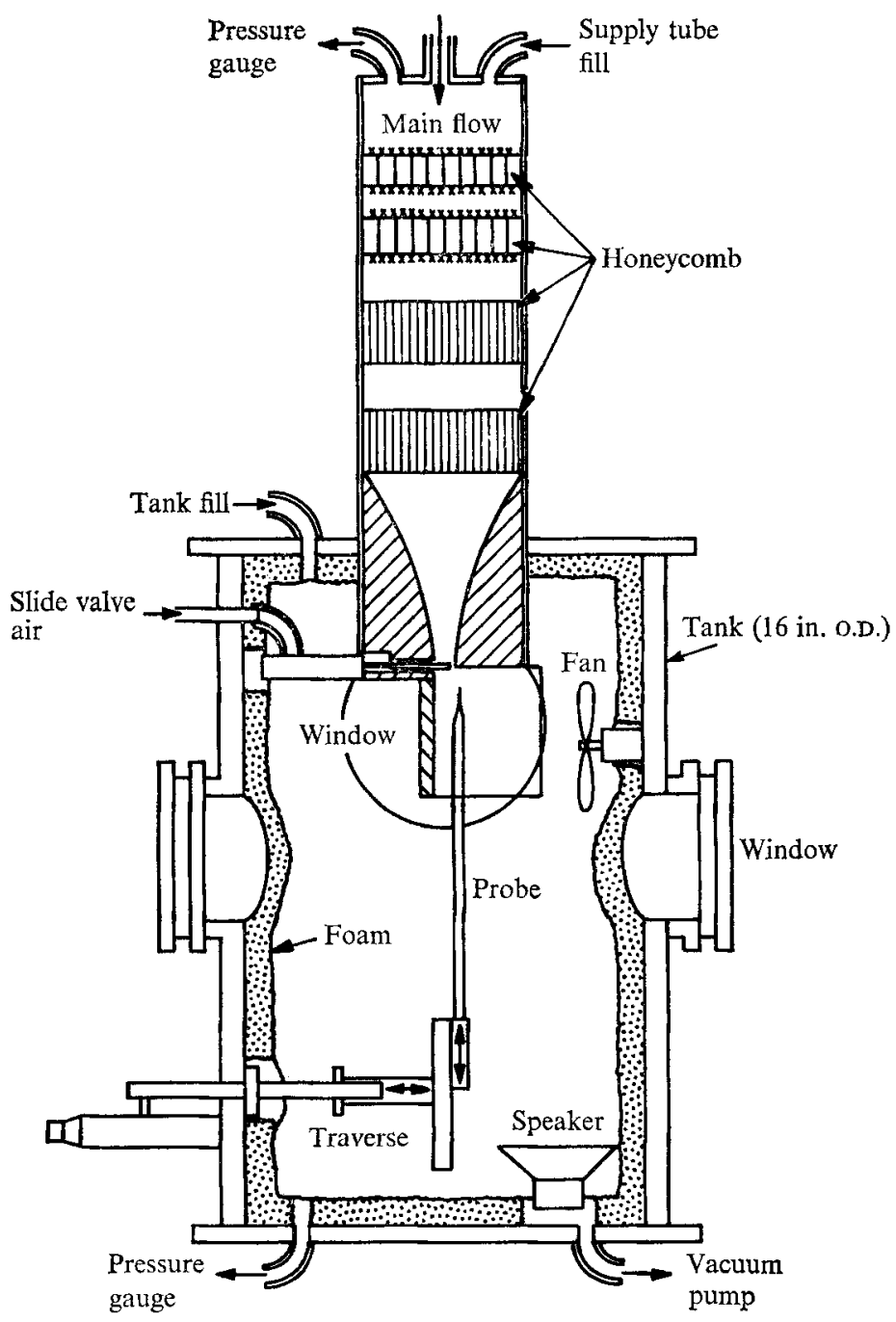

Frgure 2. Jet facility.

A loudspeaker mounted at the bottom of the tank was used to excite the shearlayer oscillations at some desired frequency. This technique has previously been used by Sato (1959), Browand (1966), Freymuth (1966) and others. Browand showed that such excitation does not change the character of the flow except for narrowing the frequency band of the disturbance oscillations. Sound forcing simplifies the analysis of the flow fluctuations by providing a known fundamental frequency and an accurate phase reference.

Gas line connexions to the tank and the settling chamber were used to evacuate the system before each run, refill the tank and settling chamber with the different freons and monitor the pressure of the two sections. 


\begin{tabular}{|c|c|c|c|}
\hline & Freon 22 & Freon 114 & Ratio: $114 / 22$ \\
\hline Molecular weight & $86 \cdot 48$ & 170.93 & 1.976 \\
\hline$k$ (joules/ms ${ }^{\circ} \mathrm{K}$ ) & $0 \cdot 01172$ & $0 \cdot 01119$ & 0.954 \\
\hline$\mu_{f}$ (poise) & $1.59 \times 10^{-4}$ & $1 \cdot 40 \times 10^{-4}$ & $0 \cdot 881$ \\
\hline$k \mid \mu_{f}^{0.45}$ & & & 0.991 \\
\hline
\end{tabular}

TABLE 1. Properties of freon 22 and freon 114 at $30^{\circ} \mathrm{C}$

\subsection{Instrumentation}

Because of the small size of the experiment (the boundary layer was about $1 \mathrm{~mm}$ thick at the separation point) and the frequency response needed for the measurement of wave parameters, sensor requirements were particularly stringent. In fact, such time response and spatial resolution could be achieved only with a single hot wire aligned spanwise to the flow. However, a single hot wire is, in general, of little value in a heterogeneous flow because the variations in the signal due to the changing fluid properties cannot be distinguished from changes due to the flow parameters that one wishes to evaluate. Although some success using two wires and mixing the signals to obtain simultaneous velocity and density measurements has been reported (e.g. Way \& Libby 1970), such probes lack the spatial resolution required for the present experiments.

Because of the many problems associated with making the desired velocity and density fluctuation measurements, a technique was adopted (Davey 1971) which allowed the measurement of mass flow rate by a single hot wire in any mixture of two particular freons, freon 22 and freon 114. The method relies upon an unusual relationship between the properties of the two gases.

The equation governing the use of hot wires is given by some form of King's law, which can be expressed as $N u=A+B R e^{n}$, where $N u$ is the Nusselt number, $R e$ is the Reynolds number, $A$ and $B$ are calibration constants and the exponent $n$ is about one half. If the relation is rewritten in dimensional form, for a given sensor operating at a fixed overheat, the hot-wire signal $e$ is related to the mass flow rates by

$$
e^{2}=A^{*} k+B *\left(k / \mu_{f}^{n}\right)(\rho u)^{n},
$$

where $A^{*}$ and $B^{*}$ are the new calibration constants, $k$ is the thermal conductivity and $\mu_{f}$ is the viscosity evaluated at the film temperature (i.e. the mean of the wire and gas temperatures). The two freons were selected because their thermal conductivities and viscosities are nearly equal, while their molecular weights are sufficiently different to produce the desired large density gradient. The properties are summarized in table 1.

Calibration tests were made to verify the theoretical predictions. The results of a test covering the range of mass flow rates used in the shear-layer experiments are shown in figure 3 . The central dashed line is a least-squares fit to all the data points. It is bounded by lines showing the predicted variation due to the small inequalities in the thermal conductivities and viscosities. The consequent uncertainty in the mass flow rate associated with a particular signal voltage varies from about $\pm 0.0031 \mathrm{~g} / \mathrm{s} \mathrm{cm}^{2}$ near the free convection limit to $\pm 0.017 \mathrm{~g} / \mathrm{s} \mathrm{cm}^{2}$ at the highest flow rates shown. These values correspond to $\pm 0.6 \%$ and $\pm 3 \%$ of 


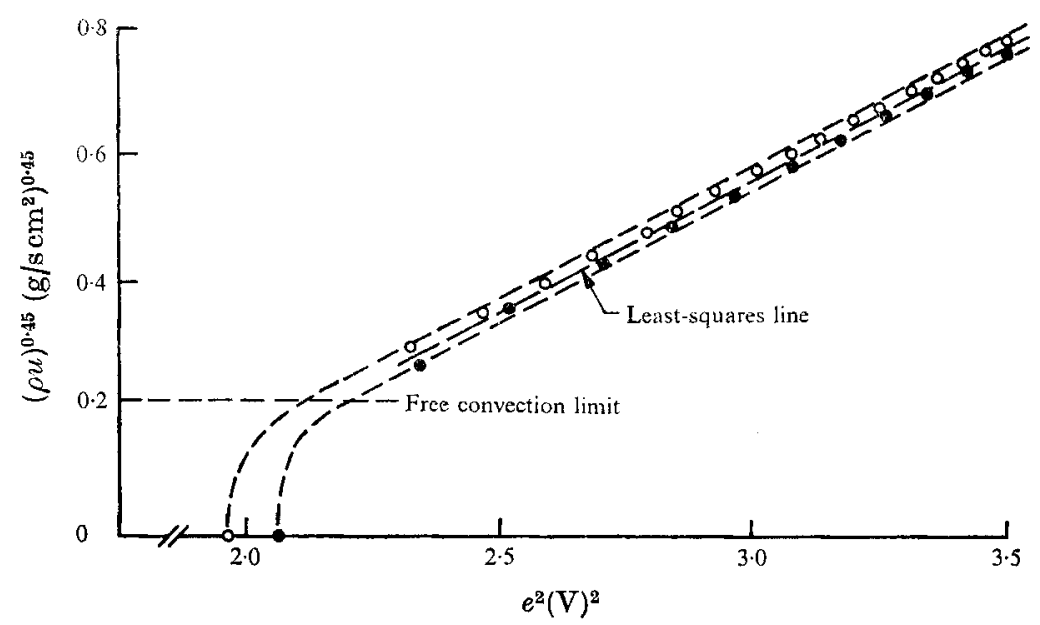

FIgURE 3. Hot-wire calibrations in freon 22 and freon 114. ○, freon 22 ; 0 , freon 114.

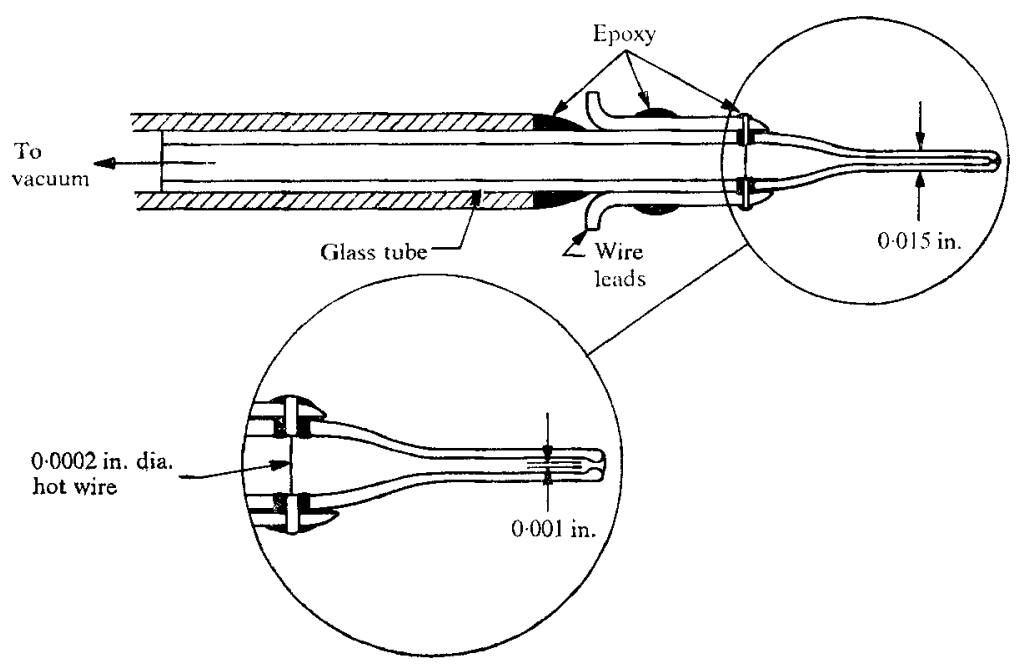

FIGURE 4. Density sensitive aspirating probe.

the experimental jet mass flow rate respectively. This inherent inaccuracy was the fundamental limitation in the experiments.

The calibration tests showed that the correct value for the power-law exponent $n$ was about $0 \cdot 45$, which is in agreement with results reported by Collis \& Williams (1959).

Measurements of mean density were made using an aspirating probe similar to those developed by Brown \& Rebollo (1972). The operation of the probe is based upon the relative insensitivity to upstream velocity of the mass flow rate through a sonic throat. For a fixed upstream pressure, the flow rate depends only upon gas properties or, in a binary mixture, only upon concentration. Since the signal from a hot wire is related to the mass flow rate over the wire it is possible to obtain a unique calibration for various mixtures of two gases by placing a hot wire inside 


\begin{tabular}{|c|c|c|c|}
\hline Experiment & 22 into 22 & 22 into 114 & 114 into 22 \\
\hline Mass flow rate $\left(\mathrm{gm} / \mathrm{s} \mathrm{cm}^{2}\right)$ & $0 \cdot 573$ & 0.580 & 0.510 \\
\hline Jet velocity $(\mathrm{cm} / \mathrm{s})$ & 336 & 335 & 150 \\
\hline Reynolds number, $U \tilde{\theta} / \nu$ & 111 & 112 & 134 \\
\hline Froude number, $U^{2} / g \tilde{\theta}$ & 一 & 4610 & 765 \\
\hline Tank pressure (psia) & 7 & 7 & 7 \\
\hline Sound forcing frequency $(\mathrm{Hz})$ & $195 \cdot 2$ & $156 \cdot 2$ & $97 \cdot 6$ \\
\hline Sound forcing amplitude (db) & 73 & 72 & 75 \\
\hline
\end{tabular}

TABLE 2. Summary of flow conditions

a tube downstream from a sonic throat. The probe design is shown in figure 4. The only significant modification of those used by Brown \& Rebollo is the use of an extended narrow neck to minimize the disturbance to the flow. Although a usable calibration was obtained the signals produced were very small, varying only about $0.05 \mathrm{~V}$ from pure freon 22 to pure freon 114 . This limited the accuracy of the measurements to about $\pm 2 \%$ of the mean density and precluded the measurement of density fluctuations.

Measurements of the sound level produced by the loud-speaker were made using a Datametries electronic manometer. The sensitivity $(0.01 \mathrm{~mm} \mathrm{Hg} / \mathrm{V})$ and frequency response were fully adequate for evaluating the $75 \mathrm{db}, 100-200 \mathrm{~Hz}$ signals used for forcing. The forcing amplitude was set high enough to ensure that the flow of oscillations was phased with the speaker signal, but not high enough to distort or significantly amplify the flow fluctuations. Hot-wire signals and an oscilloscope were used to find the proper sound level, which was about $75 \mathrm{db}$. Freymuth (1966) showed that the instability characteristies are independent of sound pressure level between 70 and $100 \mathrm{db}$.

\section{Experimental procedure}

\subsection{Selection of jet flow rates}

The desire to study a flow where viscous and buoyancy forces are negligible compared with the inertial forces produced by the density gradient led to constraints on both the Reynolds and Froude numbers. Freymuth (1966) showed that the stability characteristies of a homogeneous shear layer are independent of the Reynolds number if the Reynolds number based on the momentum thickness is greater than about sixty. However, the transition distance becomes shorter as the Reynolds number increases and, since an analysis of the downstream development of some parameters was required, practical considerations made a relatively low Reynolds number desirable. An analysis of the equations governing the stability of the heterogeneous shear layer indicated that buoyancy forces could be neglected if the Froude number was much greater than one, with $F r=U^{2} / g \theta$, where $U$ is the jet velocity and $\theta$ is the momentum thickness.

The desired combination of Reynolds and Froude numbers could be achieved with the two freons only by reducing the pressure to about half an atmosphere. This gave a Reynolds number near 100 and a Froude number greater than 750 in the worst case. The selected flow conditions are summarized in table 2. 


\subsection{Selection of running time}

Because the half-jet was exhausting into a closed reservoir, the duration of the experiment was severely restricted by the increasing static pressure and by the contamination of the background gas by the jet gas. An analysis of high-speed motion picture shadowgraphs and plots of hot-wire signals showed that relatively steady conditions were reached about $0.8 \mathrm{~s}$ after the flow was turned on and continued for about $2 \mathrm{~s}$.

The short running time made on-line data acquisition and processing impossible with the equipment available. Therefore all data signals were recorded on magnetic tape and later converted to digital form for computer processing. Three tape channels were used for hot-wire signals and a fourth was used for the loudspeaker signal. The tape was played back into a high-speed data acquisition system, which sampled the four channels simultaneously, converted the signals to digital form, multiplexed the channels, formatted the information and wrote it on digital magnetic tape. The sampling rate was set at 10000 samples per second on each channel to ensure accurate representation of the flow fluctuations.

\subsection{Analysis of flow fluctuations}

The sound forcing frequency was set at or close to the frequency of naturally occurring instability fluctuations, whose frequency had been determined by playing back recorded hot-wire signals at a reduced speed into a calibrated $x-Y$ plotter.

The digitized hot-wire signals were converted into corresponding mass flow rate measurements and then analysed with a fast Fourier transform. The program used required an input of $2^{m}$ equally spaced values and generated the amplitude and phase of $2^{m-1}-1$ harmonics of the transform fundamental. The frequency of the transform fundamental is given by $f=2^{-m} \times 10^{4} \mathrm{~Hz}$ for a digitizing rate of 10000 samples per second. As a compromise between economy and accuracy, $m$ was chosen to be ten, giving an input of 1024 samples and an output of 511 harmonics of the fundamental frequency $9 \cdot 76 \mathrm{~Hz}$. Ordinarily, this 'bandwidth' would not have provided a sufficiently accurate evaluation of the oscillation characteristics. Therefore the sound forcing frequency was set equal to one of the frequencies generated by the Fourier transform (see table 3 below). Since the natural oscillations vary by as much as ten per cent in frequency, the small differences between the chosen forcing frequencies and average natural frequencies are of no importance.

Several transform analyses were performed for each data point to make use of all available digitized measurements. The results were then averaged to give the final values.

\section{Experimental results}

\subsection{Homogeneous flow}

Experiments were first conducted using freon 22 as both the jet and reservoir gas to test the validity of the experimental and data processing procedures. Since 


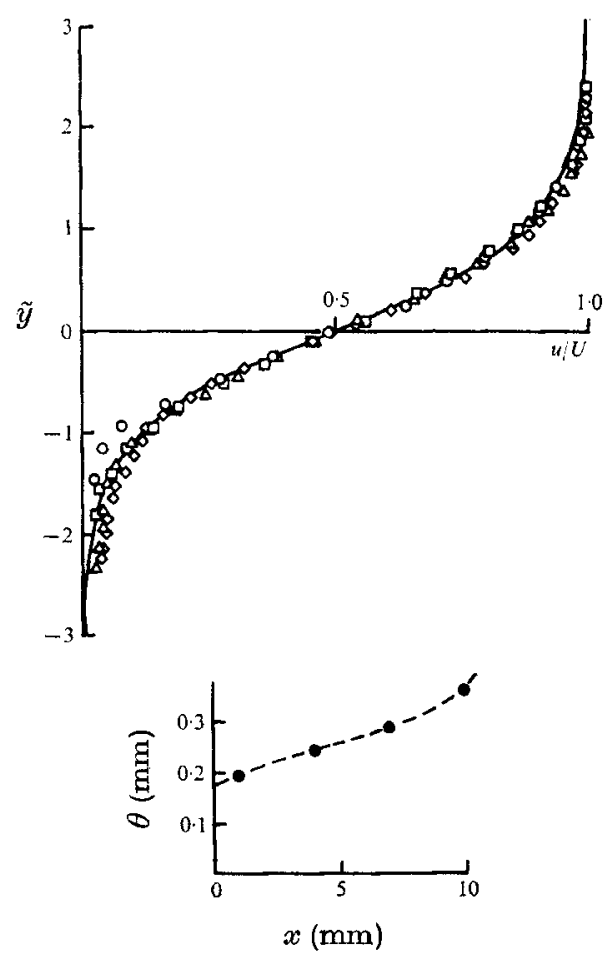

FIGURE 5. Mean velocity profiles: homogeneous flow. - $-\bar{u} / U=\frac{1}{2}(1+\tanh \tilde{y})$; $\bigcirc, x=1 \mathrm{~mm} ; \square, x=4 \mathrm{~mm} ; \Delta, x=7 \mathrm{~mm} ; \diamond, x=10 \mathrm{~mm}$.

short-duration quasi-steady experiments, digital data acquisition and processing are not the techniques usually employed for hydrodynamic stability experiments, some type of comparative analysis was needed to verify their applicability. The experimental results reported by Sato (1956), Browand (1966) and Freymuth (1966) for the homogeneous flow case provide an excellent basis for comparison.

The experiments were performed using procedures identical to those employed in the later heterogeneous flow tests. Of particular importance was the use of the same small segment of data and the same digital data processing procedures. The flow conditions (e.g. Reynolds number, tank pressure) were also the same.

Hot-wire velocity measurements were made across the shear layer at four downstream locations, all within the region of exponential disturbance oscillation growth. Mean velocity profiles were prepared and momentum thicknesses were computed. Figure 5 compares the non-dimensionalized profiles with the hyperbolic tangent profile used for theoretical analyses. The profiles have been scaled to coincide in slope and position at the point where $\bar{u} / U=0 \cdot 5$.

A reference length was needed to scale the oscillation parameters for comparison with theoretical values. Although the momentum thickness can be computed readily for any profile, the selection of a particular value to represent the entire flow is somewhat arbitrary. Theoretical models usually assume that the flow is parallel and that the momentum thickness is constant. This is not true in the experimental situation; a significant downstream increase in momentum 
thickness has been observed by Sato (1956), Browand (1966) and in the present experiments. An increase takes place just downstream from the nozzle exit as the wall boundary layer relaxes into the shear-layer profile. The developed profile then grows according to the laminar similarity law (i.e. $\theta \sim x^{\frac{1}{2}}$ ) until the transition to turbulence begins. The growth rate then increases and eventually becomes linear, as predicted by the turbulent similarity rules. Selecting a particular reference thickness is equivalent to identifying the downstream station that determines the stability characteristics of the flow. It seems evident that the reference station must lie in the region of the developed laminar shear layer. Browand chose such a reference station, lying about two-thirds of the distance between the separation point and the start of the transition to turbulence.

The present velocity profiles indicated that the reference station should lie between $x=4 \mathrm{~mm}$ and $x=7 \mathrm{~mm}$ with the corresponding thickness between 0.235 and $0.283 \mathrm{~mm}$ (figure 5). A value of $0.25 \mathrm{~mm}$ was chosen to match Browand's reference condition as closely as possible.

The frequency of the naturally occurring oscillations was determined to be about $192 \mathrm{~Hz}$, giving a value of 0.09 for the Strouhal number $S=2 \pi f \theta / U$. This is close to the values reported by Sato $(0 \cdot 10)$ and Browand $(0.092)$. Although Freymuth did not mention a natural frequency he showed that a nearly constant maximum amplification rate is attained at Strouhal numbers lying between 0.068 and $0 \cdot 136$. One would expect the naturally occurring frequency to lie in this range. Michalke (1970) reports a natural frequency non-dimensionalized in terms of $\delta_{0}^{*}$, the displacement thickness at the separation point. The present results give $f \delta_{0}^{*} / U=0.0235$, which is very close to Michalke's value, 0.023 . The sound forcing frequeney was set at $195 \cdot 2 \mathrm{~Hz}$, corresponding to the twentieth harmonic of the discrete Fourier transform used for processing. The forcing amplitude was set at $73 \mathrm{db}$.

A Fourier analysis of the velocity fluctuations was made at each point of the four profiles. Plots of the amplitude of the forcing frequency harmonic are shown in figure 6. The expected bimodal distribution across the shear layer and the increase in the amplitude downstream are evident. The plot of the amplitudes at the lower and upper peaks, $\hat{c}_{1}$ and $\hat{c}_{\mathbf{2}}$ respectively, shows that the amplification was exponential in the region surveyed. The growth rate was calculated from the slope of the peak amplitude lines using the formula

$$
-\alpha_{i}=\frac{\theta_{\text {ref }}}{x^{2}-x^{1}} \ln \frac{\hat{c}^{2}}{\hat{c}^{1}}
$$

where the superscripts refer to the two stations used. The resulting average growth rate for the two peaks was about $0 \cdot 10$. This compares reasonably well with the values $0.088,0.09$ and 0.11 reported by Freymuth, Sato and Browand respectively.

A comparison of the fluctuation amplitude distribution with experimental and theoretical results reported by Freymuth is given in figure 7. The experimental values were scaled to make the peak amplitudes match. The agreement is quite satisfactory and gratifying in light of the great differences in the experimental procedures used by Freymuth and in the present work. 


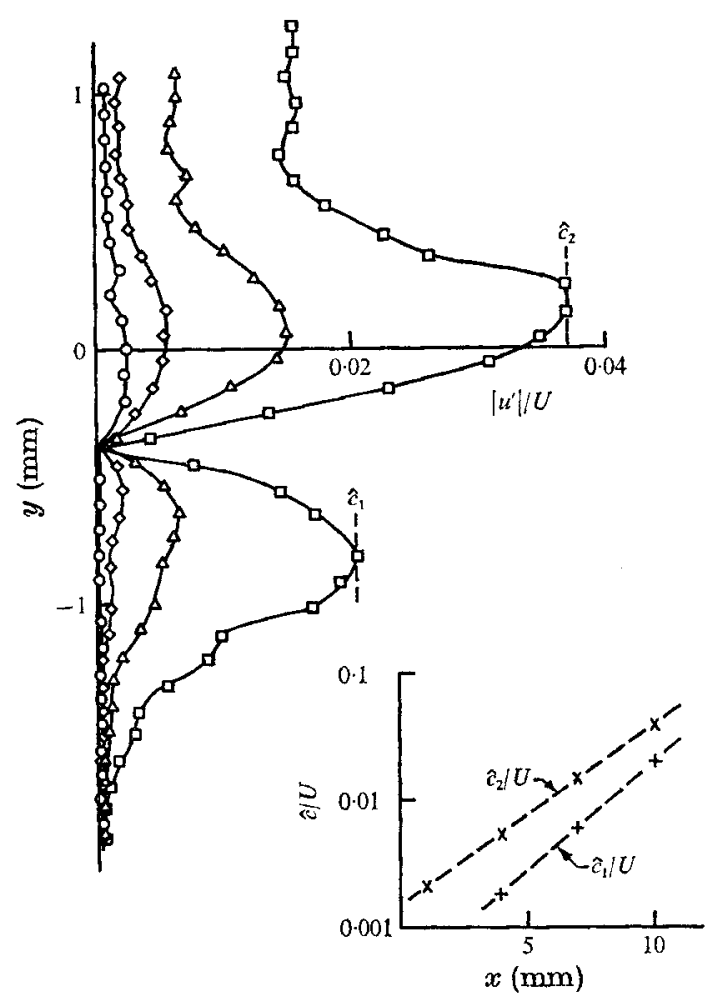

FrgURE 6. Amplitude of forcing frequency oscillations: homogeneous flow. $\bigcirc, x=1 \mathrm{~mm} ; \diamond, x=4 \mathrm{~mm} ; \triangle, x=7 \mathrm{~mm} ; \square, x=10 \mathrm{~mm}$.

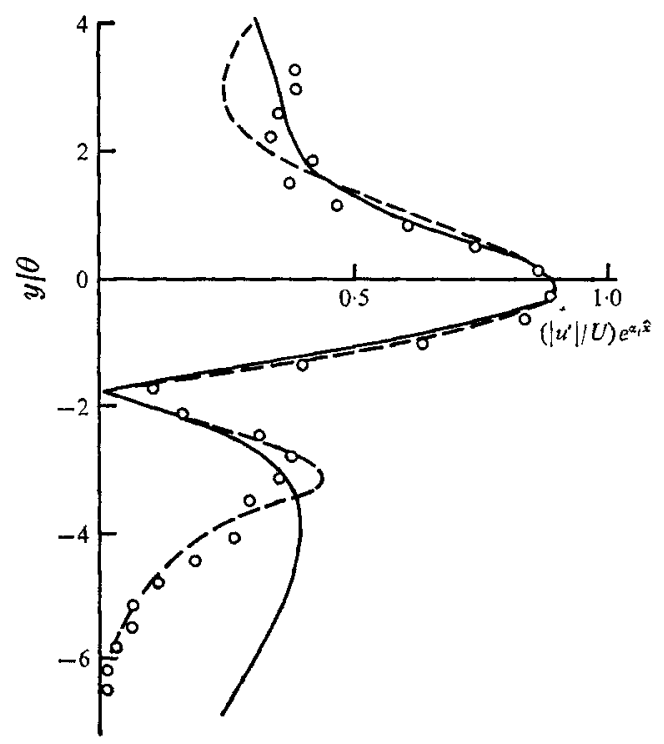

FiguRe 7. Comparison of forcing frequency oscillation amplitude measurements with results of Freymuth. Freymuth: - - theory; - - , experiment. 0 , present results, $S=0.090, x=7 \mathrm{~mm}$. 


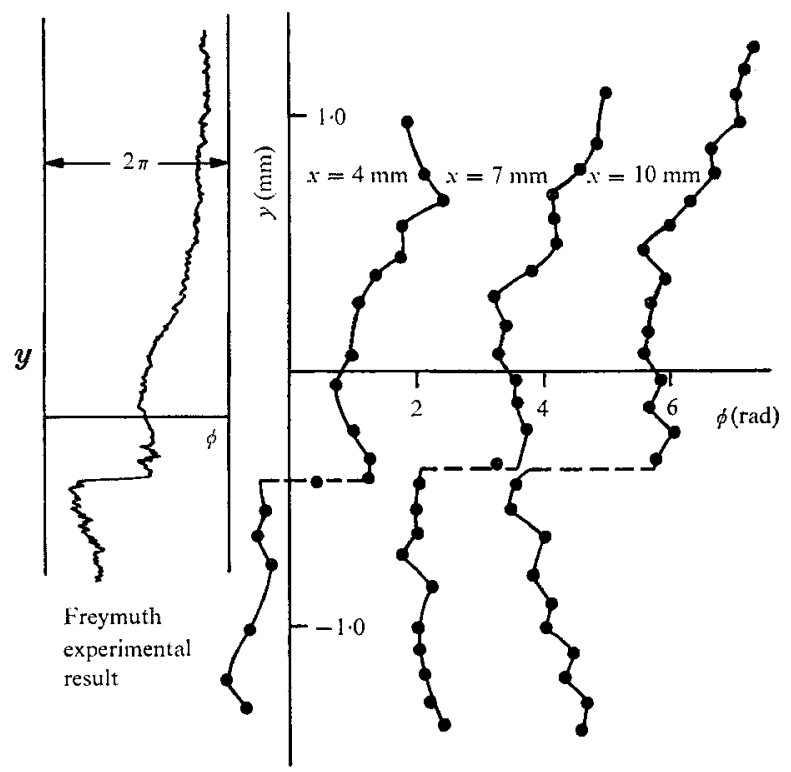

Figure 8. Phase of forcing frequency oscillations: homogeneous flow.

Phase measurements for the forcing frequency harmonic are shown in figure 8 . The phase distribution across the shear layer is seen to agree qualitatively with an unscaled plot given by Freymuth. In particular, a large change in phase is seen to occur in the low-speed portion of the profile, where the fluctuation amplitude reaches a minimum.

The wavelength of the oscillation was computed from the downstream increase in phase angle. The resulting value, $0.9 \mathrm{~mm}$, gave a value of 0.174 for the wavenumber

$$
\alpha_{r}=2 \pi \theta_{\text {res }} / \lambda \text {. }
$$

Freymuth gave a value of $0 \cdot 162$ at the corresponding Strouhal number and Browand reported a value of $0 \cdot 18$.

The agreement between the eigenvalues obtained here and those reported in previous presentations was considered satisfactory, and adequate to justify the use of the experimental procedures in heterogeneous flow experiments.

\subsection{Heterogeneous flow}

Following a development given by Baker, Rozenman \& Weinstein (1968), the hydrodynamic stability equation for a two-dimensional incompressible boundarylayer flow in a non-uniform medium can be expressed as

$$
\left(\bar{u}-\frac{\omega}{\alpha}\right)\left(\phi^{\prime \prime}-\alpha^{2} \phi\right)-\bar{u}^{\prime \prime} \phi+\frac{\bar{\rho}^{\prime}}{\bar{\rho}} \frac{\delta}{\epsilon}\left[\left(\bar{u}-\frac{\omega}{\alpha}\right) \phi^{\prime}-u^{\prime} \phi\right]=0,
$$

where the effects of viscosity, buoyancy and molecular diffusion have been neglected. In the equation, the prime indicates differentiation with respect to the transverse co-ordinate $y$, and $\phi$ is the eigenfunction generated by a Fourier decomposition of the stream function:

$$
\psi(x, y, t)=\phi(y) e^{i(\alpha x-\omega t)}
$$

The scale lengths $\delta$ and $\epsilon$ refer to the velocity and density profiles respectively. 


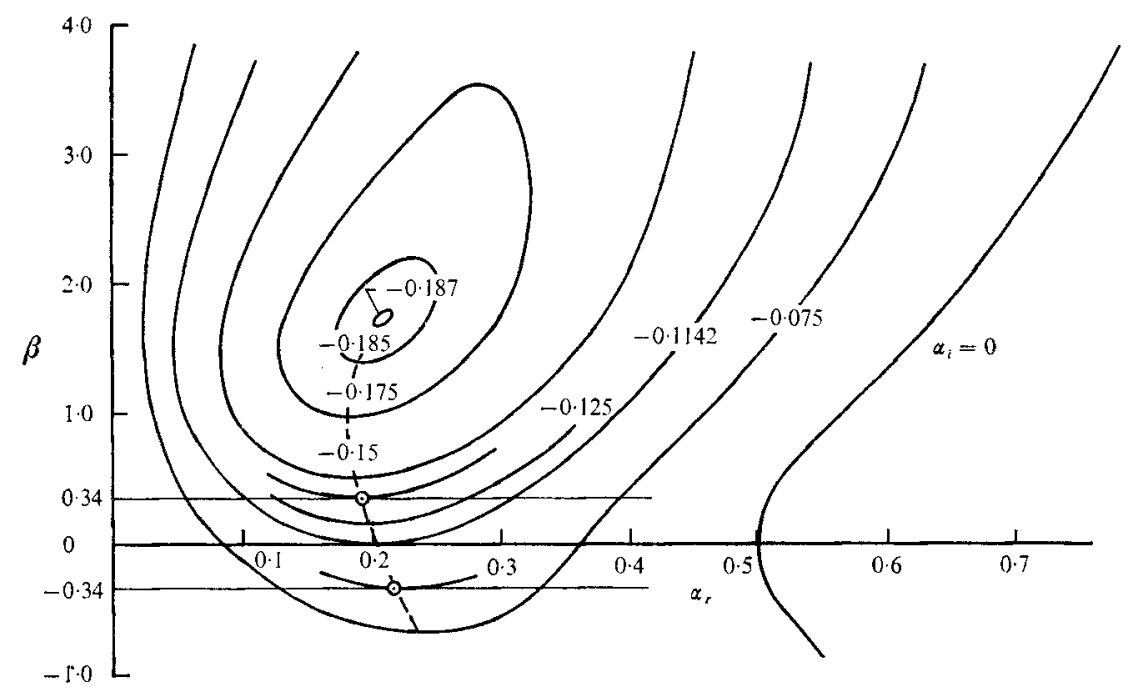

FiaURe 9. Effect of density gradient on eigenvalues, as given by Maslowe \& Kelly (1971). ---, locus of points of maximum disturbance amplification.

Maslowe \& Kelly (1971) integrated the equation using the Holmboe flow model for the mean velocity and density variations:

$$
\begin{aligned}
& \bar{u}=\frac{1}{2}(1+\tanh y), \\
& \bar{\rho}=\rho_{0} \exp \left(-\beta \tanh ^{\prime} y\right),
\end{aligned}
$$

and the free shear layer boundary conditions

$$
\phi(-\infty)=\phi(\infty)=0
$$

Although Maslowe \& Kelly calculated the stability characteristics for both temporally and spatially amplifying waves, only the latter are relevant to the experimental situation. Thus the eigenvalue $\alpha$ is taken as complex while $\omega$ is taken as real.

The calculated eigenvalues are shown graphically in figure 9 (taken from Maslowe \& Kelly 1971, figure 7). As $\beta$ increases (i.e. the flowing gas becomes lighter), the maximum disturbance amplification rate is seen to increase until it reaches an absolute maximum at $\beta=1 \cdot 7$, corresponding to a density ratio of about 30 . The wavenumber along the maximum amplification contour decreases until $\beta \simeq 0 \cdot 8$, after which it increases.

The density ratio of the freons used in the present experiments was 1.98 , corresponding to $\beta= \pm 0 \cdot 34$ for the two flow cases. Programs prepared by Maslowe were used to compute the eigenvalues and eigenfunctions at the points of maximum disturbance amplification. Experimental results are compared with these values.

Mean profiles. Average mass flow rates were computed from the digitized hot-wire signals and profiles were plotted for each downstream station. The profiles showed that a transformation from the nozzle-wall boundary-layer profile to the shear-layer profile takes place in the first few millimetres downstream from 


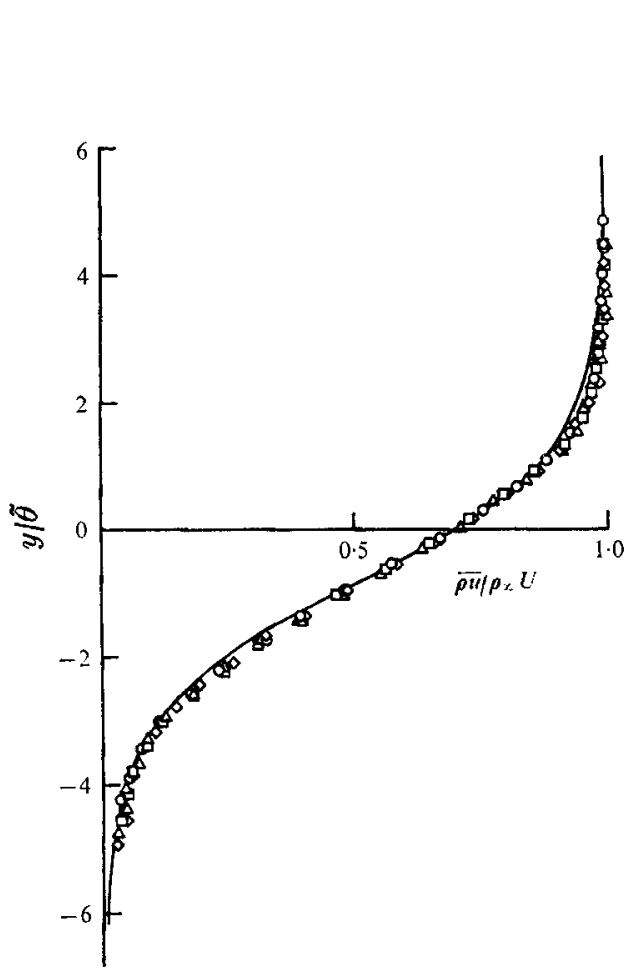

(a)

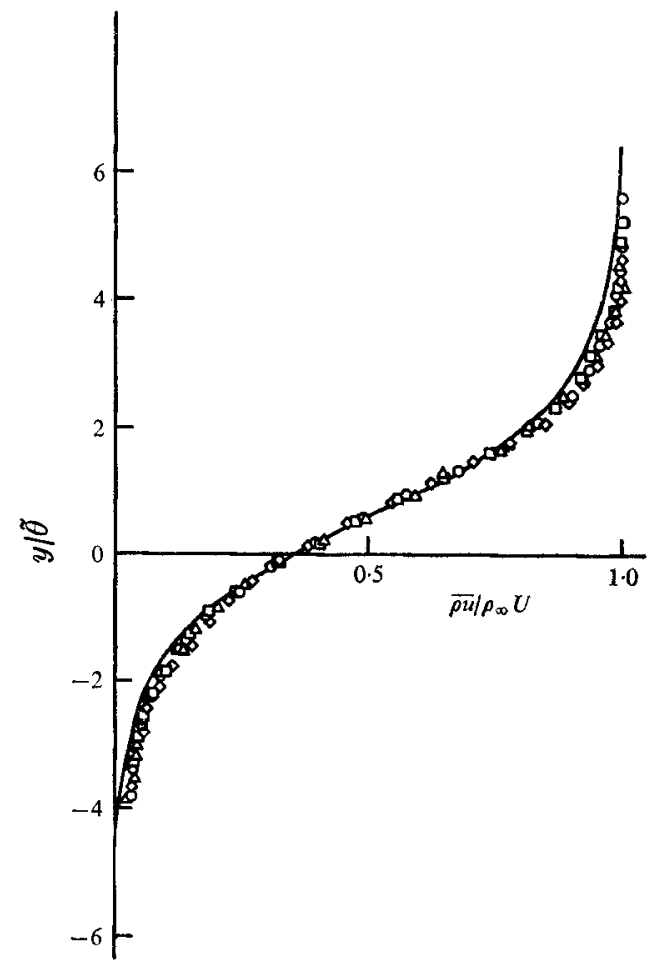

(b)

Figure 10. Mean mass flow rate profiles in developed laminar shear layer. $\overline{\rho u} / \rho_{\infty} U_{\infty}=e^{\beta} e^{-\beta \tanh v} \times \frac{1}{2}(1+\tanh y)$. (a) Freon 22 into freon 114; $0, x=4 \mathrm{~mm}$; $\square, x=5 \mathrm{~mm} ; \Delta, x=6 \mathrm{~mm} ; \diamond, x=7 \mathrm{~mm}$. (b) Freon 114 into freon $22 ; x=5 \mathrm{~mm}$; $\square, x=7 \mathrm{~mm} ; \Delta, x=9 \mathrm{~mm} ; \diamond, x=12 \mathrm{~mm}$.

the nozzle exit. Further downstream, the profile shapes remain similar for some distance. Figures $10(a)$ and $(b)$ compare these profiles with the mass flow rate profiles given by the Holmboe model:

$$
\overrightarrow{\rho u} / \rho_{\infty} U=e^{\beta} \exp (-\beta \tanh y / \tilde{\theta}) \times \frac{1}{2}(1+\tanh y / \tilde{\theta})
$$

The profile width used for scaling both the experimental and theoretical results was computed from the following integral definition based on mass flow rate values:

$$
\tilde{\theta} \equiv \int_{-\infty}^{\infty} \frac{\overline{\rho u}}{\rho_{\infty} U}\left(1-\frac{\overline{\rho u}}{\rho_{\infty} U}\right) d y
$$

This length was used in place of the more common momentum thickness because velocity values could not be determined with sufficient accuracy from the mass flow rate and density measurements. The scaled profiles were made to coincide at $\overline{\rho u} / \rho_{\infty} U=0 \cdot 5 e^{\beta}$.

Mean density profiles were plotted for stations lying within the similarity region (see figure 11). The mass flow rate thickness parameter and the adjusted origin used to scale and compare the mass flow rate profiles were used to scale the density profiles for comparison with the Holmboe model. In both flows, the scaled density profiles are considerably thinner than the model. 


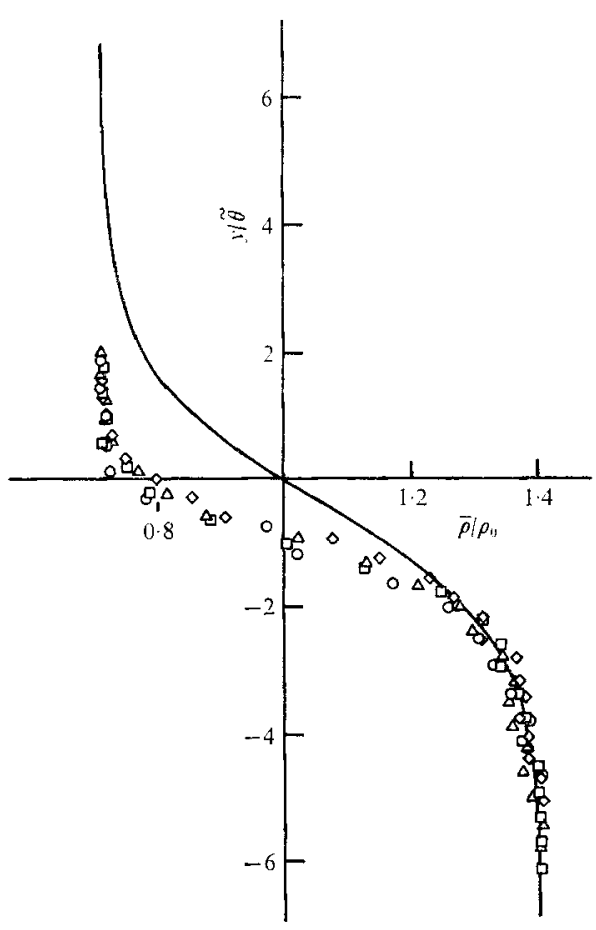

(a)

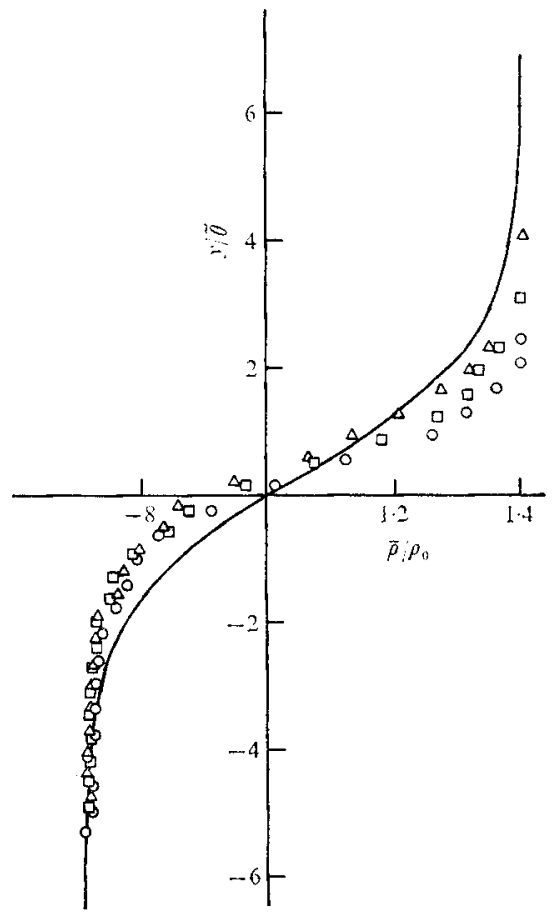

(b)

Figure 11. Mean density measurements. - $-\bar{\rho} / \rho_{0}=e^{-\beta \text { tahn } y} .(a)$ Freon 22 into freon 114, $\beta=0.34 .0, x=3 \mathrm{~mm} ; \square, x=5 \mathrm{~mm} ; \Delta, x=7 \mathrm{~mm} ; \diamond, x=9 \mathrm{~mm}$. (b) Freon 114 into freon $22, \beta=-0.34$. $O, x=5 \mathrm{~mm} \square, x=7 \mathrm{~mm} ; \Delta, x=9 \mathrm{~mm}$.

Lack of precise agreement with the Holmboe model is not too surprising since the model does not represent a theoretical solution to the steady laminar flow problem. However, the model does seem reasonable. The profile shapes have the desired asymptotic approach to free-stream and reservoir values, and the widths of the velocity and density profiles are about equal. Since the Schmidt number $S c=\nu / \mathscr{D}$ is about one for most gases, the spreading rate for equivalent velocity and density gradients should be roughly the same. Thus one would expect experimental velocity and density profiles to have about the same thickness. The disagreement in the present circumstances arises from the conditions at the nozzle exit. The velocity profile just downstream from the nozzle exit has a relatively large thickness, somewhat greater than the thickness of the wall boundary layer inside the nozzle. However, the density profile at the separation point has essentially zero thickness and its maximum gradient lies near $y=0$, in the low-speed portion of the velocity profile. Although the density profile spreads more rapidly than the velocity profile because of the higher relative gradient, its thickness does not reach that called for by the Holmboe model within the region of mass flow rate similarity. The problem is most acute when freon 22 flows into freon 114 because the similarity region is shorter. In the opposite case, the Holmboe model is approached at the portion of the region furthest downstream and one would expect better agreement with theoretical predictions based on the 
model. In either case, the higher gradients resulting from the thin density profiles should exaggerate the effects of the heterogeneity. Later results will show this to be true.

The shear-layer thickness was determined from the mass flow rate profiles and showed the anticipated downstream growth. As in the homogeneous flow results, an increase in the growth rate at some downstream location marked the beginning of the transition to turbulence. Reference thicknesses were selected at points about two-thirds of the distance from the nozzle exit to the beginning of transition, corresponding to the reference used in the homogeneous flow analysis. The resulting values, $\tilde{\theta}_{\text {ref }}=0.248 \mathrm{~mm}$ for freon 22 into freon 114 and $\tilde{\theta}=0.30 \mathrm{~mm}$ for the opposite case, were used to scale the eigenvalues for comparison with the theoretical predictions. The theoretical values were scaled using the mass flow rate thickness for the appropriate Holmboe flow model.

Analysis of flow fluctuations. The frequency of the naturally occurring flow oscillations was measured for the two flow cases. With freon 22 flowing into freon 114 the natural frequency was about $151 \mathrm{~Hz}$, giving a Strouhal number of $0 \cdot 070$. The theoretically predicted value for the maximum amplification point is $0 \cdot 076$. With freon 114 flowing into freon 22 the natural frequency varied between 90 and $117 \mathrm{~Hz}$, giving Strouhal numbers from $0 \cdot 112-0 \cdot 147$. The theoretically predicted value was $0 \cdot 129$, about midway between the measurements. The agreement between the experimental and theoretical values compares favourably with that obtained in the homogeneous case.

Profiles showing the amplitude distribution of the flow oscillation at the frequency of the sound forcing are given in figure 12. The amplitude of the peaks, evaluated as the average of the three highest point values, is plotted in figure 13 . The growth is seen to be exponential in the similarity region. Disturbance amplification rates were calculated from the slopes of the peak amplitude plots. With freon 22 flowing into freon 114 the growth rate was $0 \cdot 148$, nearly double the value obtained for the opposite flow case, $0 \cdot 076$. The significantly greater amplification rate attained with the light gas flowing into the heavier one verifies the qualitative conclusion reached from the analysis of the spark shadowgraphs and agrees with the trend predicted by Maslowe \& Kelly (1971). Eigenvalues computed with Maslowe's programs give amplification rates of 0.130 and 0.0904 for the two flow cases. In each case, the experimental results exaggerate the predicted effect of the density heterogeneity. However, all homogeneous flow results have shown that experimental amplification rates are lower than the theoretical predictions. This indicates that the fact that rate measured with freon 114 flowing into freon 22 is slightly lower than the theoretical value is an expected result. However, the excessive amplification rate observed in the opposite flow case is most unusual and demonstrates the effect of the thin density profiles and correspondingly high density gradients.

The shape of the amplitude distribution profiles is markedly different in the two flow cases. With freon 22 flowing into freon 114 , the amplitudes at the peaks are nearly equal $\left(\hat{c}_{1} / \hat{c}_{2}=0.88\right)$, whereas in the opposite flow situation the upper peak is far larger $\left(\hat{c}_{1} / \hat{c}_{2}=0 \cdot 148\right)$. Theoretical distribution shapes were prepared from the eigenfunctions calculated using Maslowe's programs. The relation for 


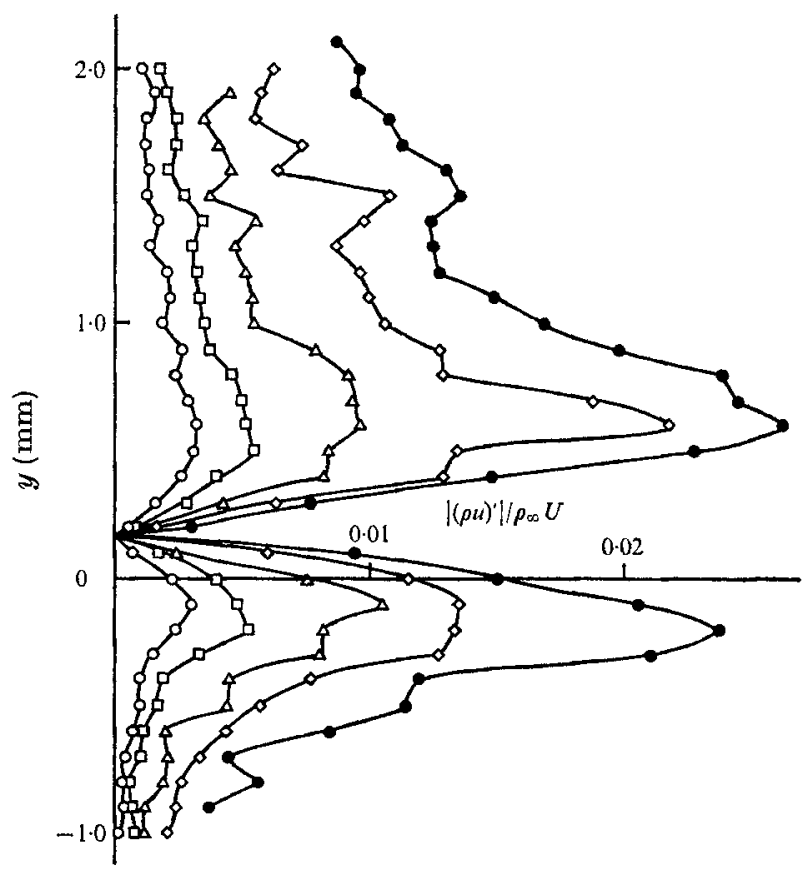

$(a)$

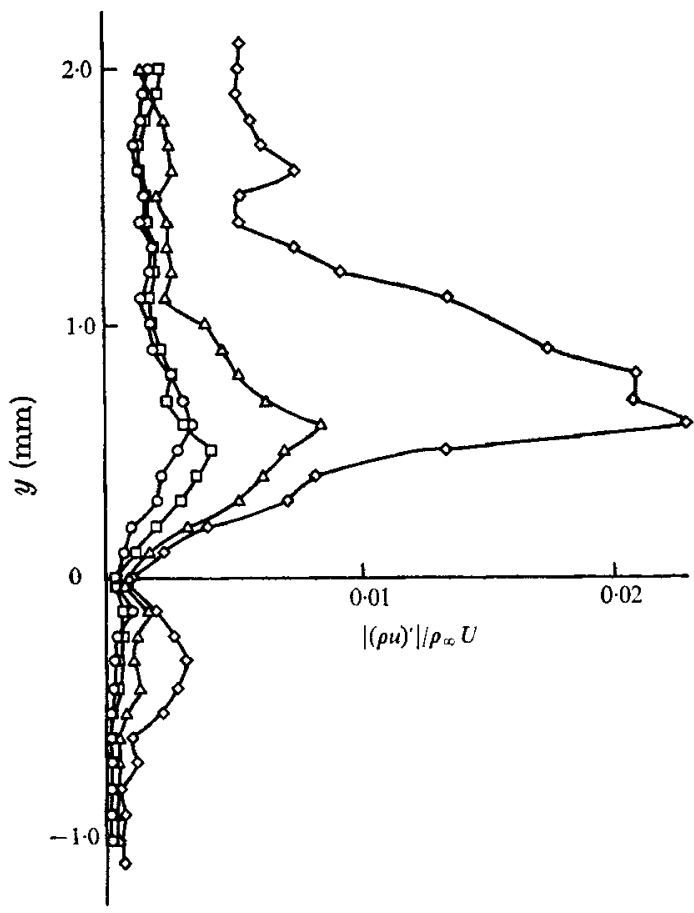

(b)

Figure 12. Amplitude of forcing frequency oscillations in the exponential growth region. (a) Freon 22 into freon $114 ; 0, x=4 \mathrm{~mm} ; \square, x=5 \mathrm{~mm} ; \Delta, x=6 \mathrm{~mm} ; \diamond, x=7 \mathrm{~mm}$; $0, x=8 \mathrm{~mm}$. (b) Freon 114 into freon $22 ; O, x=5 \mathrm{~mm} ; \square, x=7 \mathrm{~mm} ; \Delta, x=9 \mathrm{~mm}$; $\diamond, x=12 \mathrm{~mm}$. 


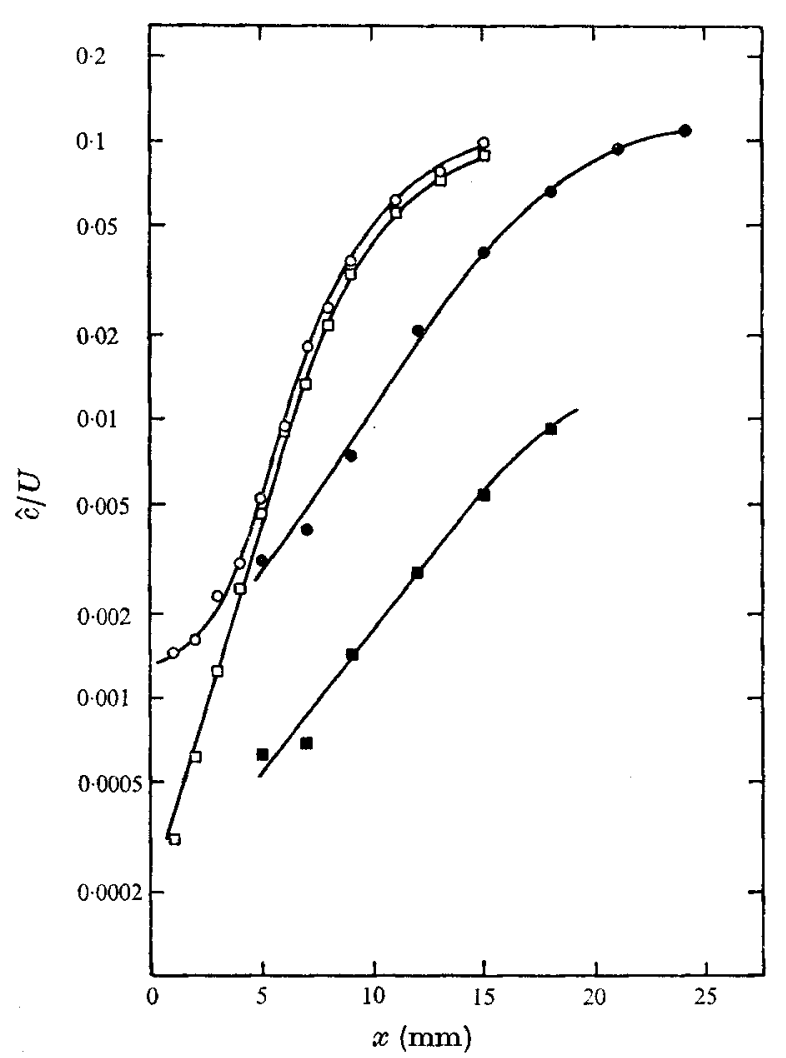

FIGURE 13. Downstream growth of peaks in the amplitude of forcing frequency oscillations. $\bigcirc, \hat{c}_{2}$, freon 22 into freon $114 ; \diamond, \hat{c}_{1}$, freon 22 into freon $114 ; 0, \hat{c}_{2}$, freon 114 into freon 22 ; $\square, \hat{c}_{1}$, freon 114 into freon 22 .

the mass flow rate fluctuation amplitude is"given by

or

$$
\begin{gathered}
\left|(\rho u)^{\prime}\right| \doteq\left|\bar{\rho} u^{\prime}+\bar{u} \rho^{\prime}\right| \\
\left|(\rho u)^{\prime}\right|=\left|\bar{\rho} \phi^{\prime}+\bar{u} \phi \frac{\alpha}{\alpha \bar{u}-\omega} \frac{d \bar{\rho}}{d y}\right| e^{\alpha_{i} x}
\end{gathered}
$$

where the mean values are given by the Holmboe model and the absolute value is computed as the modulus of the complex eigenfunction representation. Theoretical and experimental results are compared in figure 14 . The amplitudes are arbitrary, only shapes of the distributions are being compared. In both sets of profiles the amplitude of the lower peak is seen to increase steadily as $\beta$ goes from negative to positive. This effect for the mass flow fluctuations apparently does not occur in the distributions of the velocity fluctuation amplitude. The numerical calculations show no significant variation in the relative size of the peaks. This indicates that the significant term in the mass flow rate fluctuation is $\bar{\rho} u^{\prime}$. With $\beta$ negative, the mean density is greater in the upper portion of the shear layer and the relative amplitude of the upper peak would be increased. The remaining mass flow rate fluctuation term $\bar{u} \rho^{\prime}$ would be significant only if the distribution of $\left|\rho^{\prime}\right|$ varied with $\beta$. Although no experimental measurements were 


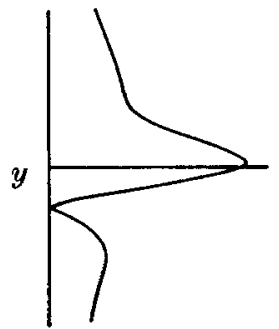

(a)

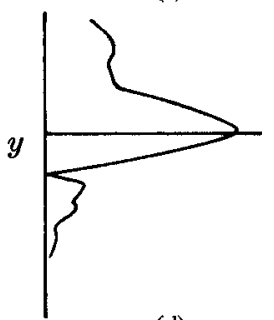

(d)

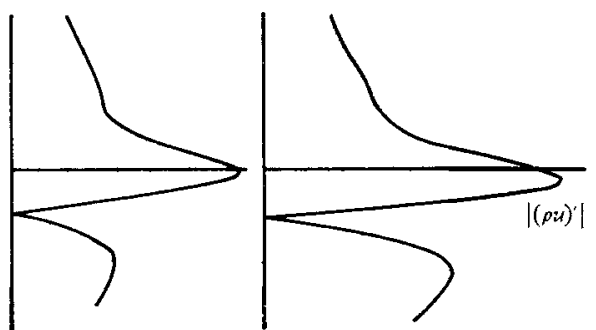

(b)

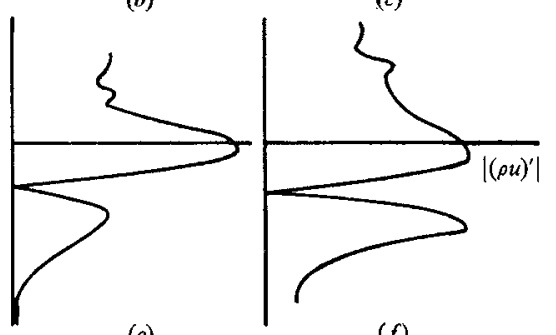

(e)

(f)

FIGURE 14. Effect of density gradient on theoretical and experimental amplitude distributions. (a) Maslowe \& Kelly, $\beta=-0 \cdot 34$. (b) Maslowe \& Kelly, $\beta=0$. (c) Maslowe \& Kelly, $\beta=0 \cdot 34$. (d) Freon 114 into freon 22. (e) Freon 22 into freon 22, homogeneous. $(f)$ Freon 22 into freon 114.

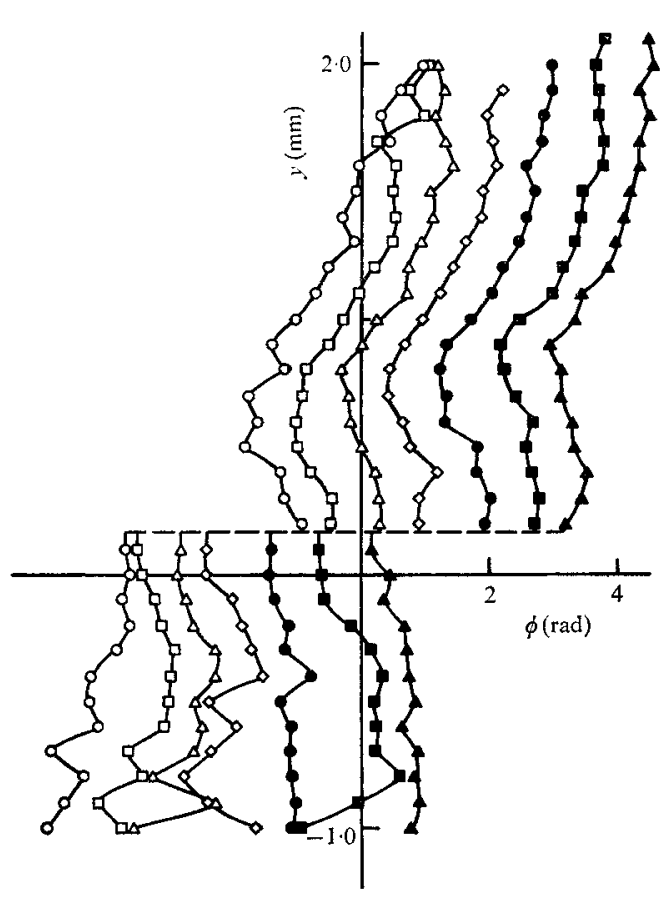

(a)

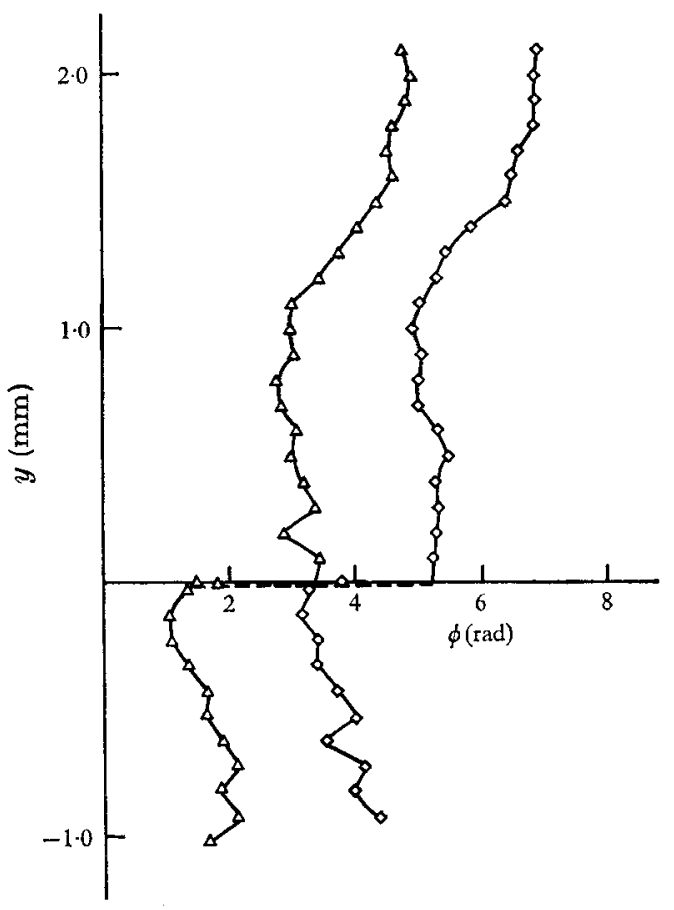

(b)

FIGURE 15. Phase of forcing frequency oscillations. (a) Freon 22 into freon $114 ; 0, x=3 \mathrm{~mm}$; $\square, x=4 \mathrm{~mm} ; \triangle, x=5 \mathrm{~mm} ; \diamond, x=6 \mathrm{~mm} ; 0, x=7 \mathrm{~mm} ; \boldsymbol{Q}, x=8 \mathrm{~mm} ; \Delta, x=9 \mathrm{~mm}$. (b) Freon 114 into freon 22; $\Delta, x=12 \mathrm{~mm} ; \diamond, x=15 \mathrm{~mm}$. 


\begin{tabular}{|c|c|c|c|c|c|c|}
\hline & \multicolumn{2}{|c|}{$\begin{array}{l}\text { Freon } 22 \text { into } \\
\text { freon } 114\end{array}$} & \multicolumn{2}{|c|}{ Homogeneous } & \multicolumn{2}{|c|}{$\begin{array}{l}\text { Freon } 114 \text { into } \\
\quad \text { freon } 22\end{array}$} \\
\hline & $\begin{array}{l}\text { Experi- } \\
\text { ment }\end{array}$ & Theory $\dagger$ & $\begin{array}{c}\text { Experi- } \\
\text { ment }\end{array}$ & Theoryt & $\begin{array}{l}\text { Experi- } \\
\text { ment }\end{array}$ & Theory $\dagger$ \\
\hline $\begin{aligned} S & =\omega_{\text {natural }} \\
& =\frac{2 \pi \tilde{\theta}_{\text {ret }} f_{\text {nat }}}{U}\end{aligned}$ & 0.0697 & 0.0761 & 0.090 & $0 \cdot 106$ & $\begin{array}{r}0.112- \\
0.147\end{array}$ & $0 \cdot 129$ \\
\hline $\begin{array}{l}\omega_{\text {forcing }} \\
\alpha_{r}=2 \pi \tilde{\theta}_{\text {ret }} / \lambda\end{array}$ & $\begin{array}{l}0.0726 \\
0.190\end{array}$ & $\overline{0 \cdot 183}$ & $\begin{array}{l}0.0915 \\
0.174\end{array}$ & $\overline{0.210}$ & $\begin{array}{l}0 \cdot 123 \\
0 \cdot 218\end{array}$ & $\overline{0.214}$ \\
\hline$\alpha_{i}=\frac{\tilde{\theta}_{\mathrm{ref}}}{x^{2}-x^{1}} \ln \frac{\hat{c}^{2}}{\hat{c}^{1}}$ & $-0 \cdot 148$ & $-0 \cdot 130$ & $-0 \cdot 10$ & -0.114 & -0.076 & -0.0904 \\
\hline $\begin{array}{l}\text { Wave speed }=\omega / \alpha_{r} \\
\hat{c}_{1} / \hat{c}_{2}\end{array}$ & $\begin{array}{l}0.382 \\
0.88\end{array}$ & $\begin{array}{l}0.416 \\
0.626\end{array}$ & $\begin{array}{l}0.526 \\
0.35\end{array}$ & $\begin{array}{l}0.506 \\
0.453\end{array}$ & $\begin{array}{l}0.564 \\
0.148\end{array}$ & $\begin{array}{l}0.603 \\
0.272\end{array}$ \\
\hline
\end{tabular}

TABLE 3. Comparison of experimental and theoretical eigenvalues and eigenfunctions for various flow cases

$\dagger$ Theoretical eigenvalues and eigenfunctions evaluated at the point of maximum disturbance amplification rate and scaled with the mass flow rate profile thickness of the Holmboe model using programs prepared by Maslowe.

made, the theoretical distributions are unimodal in both flow cases, with the peak near the centre of the shear layer. Thus this term would not affect the shape of the mass flow rate fluctuation distribution.

Plots of the phase distribution across the shear layer are given in figures $15(a)$ and $(b)$ for the two flow cases. As for the homogeneous flow results, a large phase shift is evident in the low-speed portion of the shear layer at the point where the fluctuation amplitude reaches a sharp minimum. Wavelengths were determined from the downstream increase in phase angle. With freon 22 flowing into freon 114 the wavelength was $0.82 \mathrm{~cm}$, giving a wavenumber of 0.190 , which is close to the theoretical value of 0.183 . In the opposite flow case, the wavelength was $0.864 \mathrm{~cm}$ and the experimental and theoretical wavenumbers were $0 \cdot 218$ and $0 \cdot 214$ respectively. A summary of all experimental and comparative theoretical eigenvalues is given in table 3 .

\section{Remarks}

The reasonably close agreement between the measured and theoretical eigenvalues and eigenfunctions suggests that the hydrodynamic stability analysis performed by Maslowe \& Kelly adequately describes the mechanics of the flow. The differences between the calculations and the measurements are, at least qualitatively, such as to be expected from the differences in the experimental and the model profiles. We conclude that the effects of viscosity, buoyancy and molecular diffusion can indeed be neglected in comparison with the inertial forces produced by the density gradient. The instability mechanism is basically kinematic and the amplification is due to the same type of vortex induction as that which leads to the instability of a vortex sheet. (However, a quantitative 
description of vortex motion in a non-uniform density field is not as simple as for the incompressible case.)

The most interesting finding of both the experimental and theoretical analyses is the increased amplification rate associated with a negative density gradient. Although the phenomenon can be calculated theoretically, it is not readily explained in more physical terms. The basic vortex sheet instability follows from the change in vorticity distribution induced by a sinusoidal displacement of the sheet. The resulting perturbation velocities are equivalent to a concentration of vorticity at points where the displacement slope is negative. The added vorticity then increases the displacement of the sheet which further concentrates the vorticity, and so on. How this process is altered by a transverse density gradient is not obvious.

The effect of different densities on the two sides is readily incorporated into the stability calculation of a vortex sheet (e.g. see Landau \& Lifshitz 1959). The temporal amplification rate is

$$
-\omega_{i}=k U \frac{\left(\rho_{1} / \rho_{2}\right)^{\frac{1}{2}}}{\rho_{1} / \rho_{2}+1}=\frac{k U\left(\rho_{2} / \rho_{1}\right)^{\frac{1}{2}}}{\rho_{2} / \rho_{1}+1} \leqslant k U,
$$

that is, the two sides of the sheet are indistinguishable, as follows from a Galilean transformation principle, and the effect of any density difference is stabilizing. The same analysis shows that for a spatially amplifying wave the amplification rate is

$$
-\alpha_{i}=(\omega / U)\left(\rho_{1} / \rho_{2}\right)^{\frac{1}{2}}
$$

where $\rho_{1}$ corresponds to the side of the jet where the velocity is zero and $\rho_{2}$ corresponds to the high-speed side, with velocity $U$. Thus, for $\rho_{2} / \rho_{1}<1$, the spatial amplification rate is increased. This is qualitatively in agreement with the calculated and experimental results, but for shear layers of finite thickness the dependence on $\rho_{2} / \rho_{1}$ is to a power of about $0 \cdot 3$ rather than $\frac{1}{2}$.

As has been pointed out by many writers in recent years, temporally growing disturbances cannot be transformed linearly into spatial disturbances. The above example demonstrates this rather forcefully. Considering the magnitude of the amplification rates (the amplitude is doubled in one seventh of a wavelength in our example of freon 22 flowing into freon 114), it is not surprising that timedependent and spatially dependent growth rates are not simply transformable.

The authors are grateful to D. E. Coles for his advice on various aspects of the experiment and to the Office of Naval Research for support under Contract N14 67A94 001.

\section{REFERENCES}

Baker, R. L., Rozenman, T. \& WeInstein, H. 1968 Stability of shear flow with density gradient and viscosity. N.A.S.A. Contractor Rep. CR-958.

BrowaNd, F. K. 1966 An experimental investigation of the instability of an incompressible, separated shear layer. J. Fluid Mech. 26, 281.

Brown, G. L. \& Rebollo, M. 1972 A probe to measure density. A.I.A.A. J. To be published. 
Coliss, D. C. \& WrLframs, M. J. 1959 Two-dimensional convection from heated wires at low Reynolds numbers. J. Fluid Mech. 6, 357.

DaveY, R. F. 1971 Mass flow rate measurements in a heterogeneous medium A.I.A.A.J. 9, 1874.

Freymuth, P. 1966 On transition in a separated laminar boundary layer. J. Fluid Mech. 25,683 .

GROPHNGIESSER, H. 1969 Beitrag zur Stabilität freier Grenzschichten in kompressiblen Medien. Deutsche Luftund Raumfahrt, Forsch.-Ber. 69-25.

Landau, L. D. \& Limswrm, E. M. 1959 Fluid Mechanics. Addison-Wesley.

MasLowe, S. A. \& Krelcy, R. E. 1971 Inviscid instability of an unbounded heterogeneous shear layer. J. Fluid Mech. 48, 405.

MICHALKE, A. 1970 The instability of free shear layers: a survey of the state of art. Deutsche Luft-und Raumfahrt, Mitt. 70-04.

SATO, H. 1956 Experimental investigation on the transition of a laminar separated layer. J. Phys. Soc. Japan, 11, 702.

SATo, H. 1959 Further investigation on the transition of two-dimensional separated layer at subsonic speeds. J. Phys. Soc. Japan, 14, 1797.

WAY, J. \& LIBBY, P. 1970 Hot-wire probes for measuring velocity and concentration in helium-air mixtures. A.I.A.A. J.8, 976. 

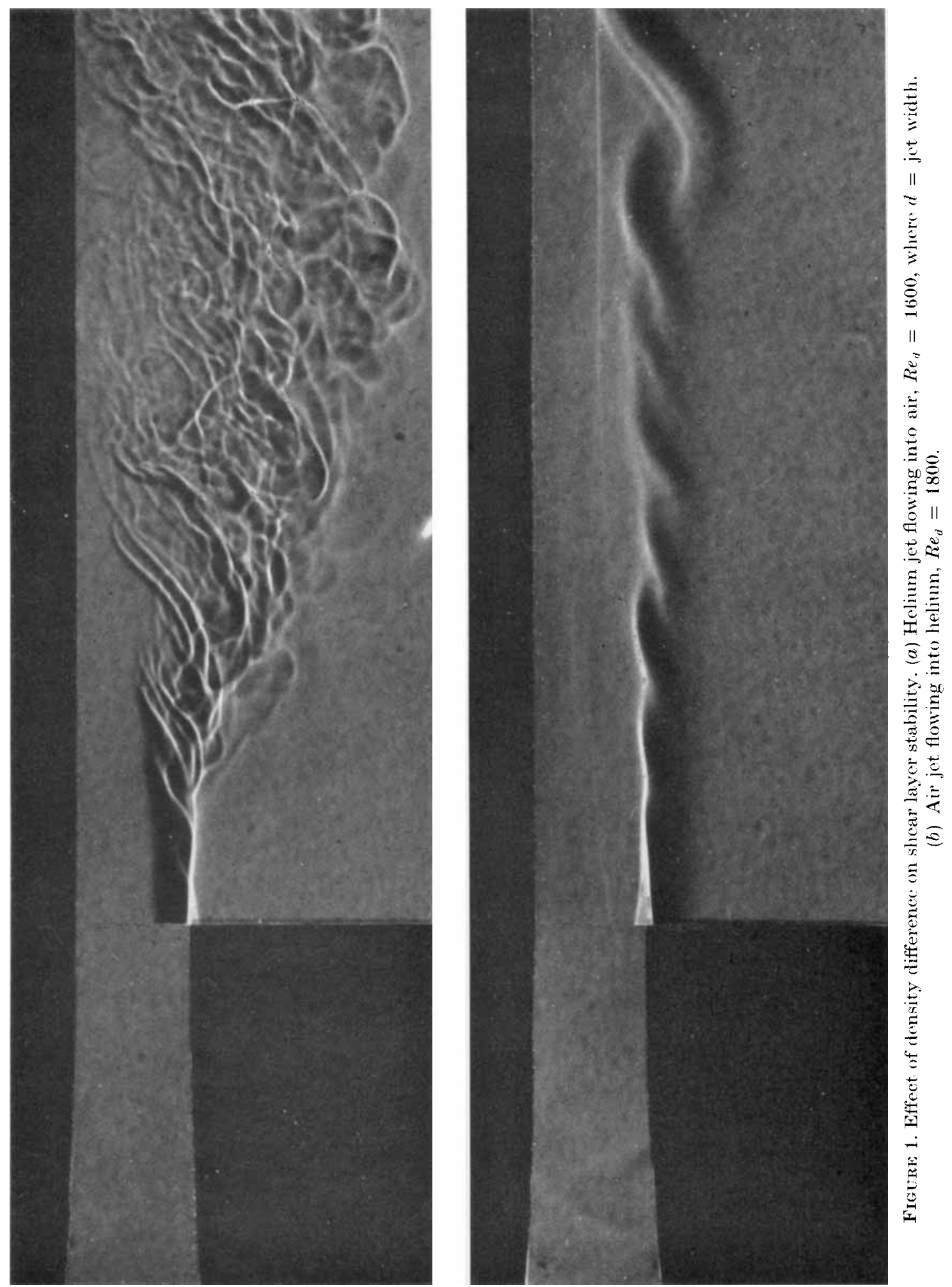